\title{
RELAÇÃO ENTRE A INTEGRAÇÃO SENSORIAL E O MÉTODO PADOVAN®
}

\author{
Relationship Between Sensory Integration And Padovan ${ }^{\circledR}$ Method
}

Lilianny Pereira ${ }^{1 *}$, Gislei Frota Aragão², Daianny Mesquita Ponte ${ }^{1}$, Adrielle Silva Alencar ${ }^{1}$, Ruan Lucas Romão ${ }^{1}$

\begin{abstract}
RESUMO
Objetivo: revisão de literatura sobre integração sensorial e o Método Padovan®, correlacionando-os a fim de demonstrar como o Método trabalha dentro desta abordagem de estimulação sensório-motora e, assim, analisar os benefícios dessa terapia. Fontes de dados: Foi utilizada as seguintes bases de dados: Medline, Scielo, Periódico CAPES e LILACS. Foram selecionados artigos publicados entre o período de 2010 a 2021 e incluídos 6 artigos publicados sobre o Método Padovan ${ }^{\circledR}$ fornecidos pelos próprios autores. Os descritores usados foram: Integração sensorial, neuroplasticidade, desenvolvimento infantil e terapia ocupacional com todas as combinações possíveis entre eles. Todos os artigos selecionados foram publicados na língua inglesa. Síntese dos dados: A busca forneceu 2631 estudos sobre integração sensorial. A partir destes, foram selecionados 347 artigos da Medline, 6 da Scielo, 926 do portal CAPES, 302 da LILACS, além dos 6 sobre o Método Padovan ${ }^{\circledR}$ cedidos pelos autores. Após critérios de inclusão e exclusão 34 artigos foram analisados na sua íntegra e, após cruzadas as informações sobre integração sensorial e a Terapia Padovan®, viu-se que a mesma tem em sua constituição os fundamentos da estimulação sensório-motora. Conclusão: Estímulos sensoriais externos são fundamentais para o desenvolvimento do Sistema Nervoso Central. Assim o Método Padovan ${ }^{\circledR}$, terapia embasada na integração sensorial, que atua através da neuroplasticidade, pode ser útil para tratar distúrbios do neurodesenvolvimento e oferecer melhorias na qualidade de vida aos pacientes.
\end{abstract}

Palavras-chave: Integração sensorial, Neuroplasticidade, Desenvolvimento infantil e Terapia ocupacional;

\begin{abstract}
Objective: to review the literature on sensory integration and the Padovan Method®, correlating them in order to demonstrate how the Method works within this sensory-motor stimulation approach and, thus, to analyze the benefits of this therapy. Data sources: Literature review using the following databases: Medline, Scielo, Periodic CAPES and LILACS. Articles published between 2010 and 2021 were selected and 6 articles published on the Padovan Method® were included and provided by the authors themselves. The descriptors used were: Sensory integration, neuroplasticity, child development and occupational therapy with all possible combinations between them. All selected articles were published in English. Summary of the findings: The search provided 2631 studies on sensory integration. From these, 347 articles from Medline, 6 from Scielo, 926 from CAPES portal, 302 from LILACS were selected, in addition to the 6 on the Padovan Method® provided by the authors. After inclusion and exclusion criteria 34 articles were analyzed in their entirety and, after crossing the information on sensory integration and Padovan $®$ Therapy, it was seen that it has in its constitution the fundamentals of sensorimotor stimulation. Conclusion: External sensory stimuli are essential for the development of the Central Nervous System. Thus, the Padovan Method® ${ }^{\circledR}$, therapy based on sensory integration, which works through neuroplasticity, can be useful to treat neurodevelopmental disorders and offer improvements in the quality of life for patients.

Keywords: Sensory Integration, Neuroplasticity, Child development and Occupational Therapy.
\end{abstract}

1. Faculdade de Medicina Estácio de Juazeiro do Norte.

2. Universidade Estadual do Ceará.

*Autor para Correspondência. E-mail: liliannypediatra@hotmail.com 


\section{INTRODUÇÃO}

Inicialmente pensava-se que o cérebro era uma estrutura rígida sem capacidade de mudança, pois, sabe-se que permanece constantemente na interfase, ou seja, fase do ciclo celular em que não ocorre replicação celular, assim, lesões em potencial não poderiam ser restauradas ${ }^{1}$. Atualmente, há evidências que provam que o tecido nervoso é capaz de se adaptar a várias circunstâncias durante toda a vida, sendo válido dizer que o cérebro é flexível e modificável $^{2}$. Esta capacidade é chamada de neuroplasticidade, onde há um rearranjo das conexões interneuronais baseadas em novas experiências vividas durante o ciclo celular com modificações moleculares e estruturais como a formação de novas sinapses neuronais ${ }^{3}$. O objetivo desta complexa modificação é a aquisição comportamental de marcos motores e não-motores do desenvolvimento via aprendizagem e memória ${ }^{4}$.

A neuroplasticidade é o potencial do sistema nervoso de agrupar e organizar os circuitos neurais a fim de manter o desempenho excelente e efetivo das funções sensóriomotoras. A partir dessa capacidade, a integração sensorial, como um processo neurofisiológico imprescindível, organiza as informações aferentes e as compara à experiência cerebral preexistente, garantindo as respostas adequadas diante de qualquer estímulo ${ }^{5}$. Esse processo, ao desenvolver um padrão motor e sensitivo, trabalha os dispositivos visuais, auditivos e táteis de forma controlada para que, conforme a aplicação repetitiva de estímulos, as sinapses neurais se estabeleçam e sejam integradas corretamente 6 . Um modelo de terapia ocupacional baseado na integração sensorial, além de ser eficaz em reformar as habilidades de processamento, se torna significativo por facilitar a assimilação das informações sensoriais, e pela capacidade de fornecer a base para o desenvolvimento de uma certa independência no cotidiano ${ }^{7}$.

A teoria de integração sensorial desenvolvida por Jean Ayres, baseada nos princípios da neurociência, reforçada pelos avanços científicos e aprimorada pela prática clínica, estabelece as relações entre mecanismos de recepção neural, modulação de informações de entrada e integração para culminar com uma resposta de saída resultando, finalmente, em uma adaptação comportamental. À luz da plasticidade neuronal é possível compreender esta capacidade adaptativa do sistema nervoso, cujo desenvolvimento sensório-motor condiciona o processo de aprendizagem, aliado às interações com o meio externo que contribuem para o desenvolvimento cerebral e para a aquisição de habilidades. Através dessa teoria é possível estabelecer a importância do processo integrativo para o desempenho corporal pois diante dos estímulos do meio ambiente, atividades em âmbito motor e sensitivo podem mediar a neuroplasticidade, fortalecendo a base da abordagem utilizada pelas terapias fundamentadas na integração sensorial ${ }^{7,8,9}$.

O Método Padovan ${ }^{\circledR}$, criado por Beatriz Padovan na década de 70, apresenta uma modalidade de terapia que se baseia na neuroplasticidade, pois promove uma série de exercícios que reproduzem movimentos próprios do desenvolvimento ontogenético humano que torna o homem apto a pôr-se de pé e andar, assim como desenvolver suas capacidades na linguagem e pensamento, principalmente pelos exercícios para funções reflexo-vegetativas orais (respiração-sucção-mastigação-deglutição) ${ }^{10,11,12,13}$. Esse método atua com todas as funções em conjunto, visto que os reflexos primitivos são interligados e seguem uma ordem, sendo necessário trabalhá-los de forma integrada e na sequência de seu desenvolvimento, para que possam ser executados de forma correta pelo terapeuta ou paciente.

Logo, a partir do Método Padovan a integração sensorial é desenvolvida através do estímulo conjunto dos sentidos pelos seus exercícios que são acompanhados com música e trabalham habilidades visuais, motoras, táteis e auditivas, consistindo em um método natural que desfruta puramente da fisiologia ${ }^{11,12}$.

Diante desse cenário, o objetivo do presente artigo é fazer uma revisão sobre os efeitos da Integração Sensorial nos processos cerebrais e sua utilização em técnicas terapêuticas e correlacionar os exercícios do Método Padovan com a integração sensorial, descrevendo os exercícios que compõem essa terapia e possibilitam tal adaptação, bem como analisar os benefícios alcançados através desse tratamento. Além disso, os poucos trabalhos científicos sobre o método em questão e as baixas opções de terapias que trabalhem a integração sensorial sustentam a relevância e a necessidade de trabalhos como este.

\section{METODOLOGIA}

Trata-se de uma revisão de literatura do tipo narrativa, onde foi realizada a pesquisa e busca dos artigos nas seguintes bases de dados: Medline (PubMed), Portal Periódico CAPES, Scielo (Biblioteca Virtual em Saúde) e Literatura Latino-Americana e do Caribe em Ciências da Saúde (LILACS). Para a abordagem do Método Padovan ${ }^{\circledR}$ foi utilizado artigos publicados pelos autores deste estudo e outros disponibilizados no site do Método Padovan ${ }^{\circledR}$. Os descritores usados foram integração sensorial, neuroplasticidade, desenvolvimento infantil e terapia ocupacional, utilizados também na língua inglesa e cruzado 
os termos entre si. Os artigos escolhidos seguiram a linha do tempo de período de 12 anos, datando de 2010 a 2021, à exceção do artigo original do Método Padovan, que é de 1997, e que fossem publicados em inglês, português ou espanhol, sendo incluso qualquer tipo de artigo que tivesse como participantes somente humanos, e que utilizassem a terapia de integração sensorial.

A seleção dos artigos foi feita pela leitura prévia dos resumos seguindo os seguintes critérios de exclusão: artigos que fugiam da abordagem central, que se restringiam a um dos 5 sentidos humanos (visão, audição, tato, paladar e olfato), estudos com animais ou que abordava somente controle postural e equilíbrio. As comparações que foram feitas entre os dois métodos (Integração Sensorial e Método Pandovan ${ }^{\circledR}$ ) tinham como foco os benefícios à população em estudo, uso da neuroplasticidade e semelhanças dos exercícios utilizados nas terapias em estudo.

Para avaliar os riscos de viés dos artigos selecionados os autores basearam nos seguintes padrões: Se havia critérios de inclusão e exclusão pré-definidos nos estudos, se as pesquisas foram consistentes, se os resultados são precisos e aplicáveis na prática. Esta etapa foi realizada por dois autores que fizeram estas avaliações, caso houvesse discrepância um terceiro autor seria acionado para avaliação final do artigo. Durante a leitura dos artigos selecionados foram definidos pontos importantes como: características da população em estudo, patologias apresentadas pela população, principais resultados e aos dados dos estudos observacionais e revisões disponíveis foi realizado uma síntese narrativa. Todas estas principais informações foram organizadas em tabela.

\section{RESULTADOS}

A busca preliminar dos artigos forneceu 2631 estudos, dos quais foram selecionados inicialmente 347 da Medline, 6 da Scielo, 926 do Portal CAPES, 302 da LILACS e inclusos 6 artigos publicados pelos autores deste trabalho. Foram excluídos 2584 artigos após aplicados os critérios de inclusão e exclusão. Por fim, 34 artigos foram selecionados para compor este estudo. Destes, 9 foram revisões de literatura, 8 revisões sistemáticas, 10 estudos experimentais, 3 observacionais, 2 relatos de casos e 2 editoriais. A grande disparidade entre o número de estudos localizados e o número de publicações dos selecionados deve-se à escassez de trabalhos a respeito do Método Padovan e Terapia de Integração Sensorial em humanos.

Os estudos experimentais foram detalhados na Tabela 1 de acordo com a população avaliada e suas respectivas patologias, a fim de apresentar o efeito que a terapia de integração exerce sobre uma população de crianças e quais déficits respondem aos estímulos empregados.

Tabela 1: Informações referentes as patologias cujos métodos foram aplicados e a população utilizada em cada estudo.

\begin{tabular}{|c|c|c|}
\hline Estudo & População & Patologias \\
\hline $\begin{array}{l}\text { SANG HJ, JUNG-HO L, } \\
2016^{14}\end{array}$ & $\begin{array}{l}\text { Adultos (média de } 65 \\
\text { anos) }\end{array}$ & $\begin{array}{l}\text { Acidente vascular } \\
\text { encefálico (AVE) }\end{array}$ \\
\hline PAVÃO SL, $2016^{15}$ & Crianças de 3 a 15 anos & Paralisia cerebral \\
\hline ETIN SP, $2011^{16}$ & $\begin{array}{l}\text { Recém-nascidos } \\
\text { prematuros }\end{array}$ & Prematuridade \\
\hline WEINSTEIN M, $2015^{17}$ & Crianças de 0 a 7 anos & Hemiparesia \\
\hline $\begin{array}{l}\text { PERUZOLLO DL,2017 } \\
18\end{array}$ & Crianças de 0 a 7 anos & $\begin{array}{l}\text { Transtorno do } \\
\text { Espectro Autista } \\
\text { (TEA) }\end{array}$ \\
\hline $\begin{array}{l}\text { IWANAGA R et al, } 2013 \\
\text { I9 }\end{array}$ & ( 20 crianças - 2 a 6 anos) & $\begin{array}{l}\text { Transtorno do } \\
\text { Espectro Autista } \\
\text { (TEA) }\end{array}$ \\
\hline $\begin{array}{l}\text { MOHAMMED AH, } \\
2015^{20}\end{array}$ & (34 crianças - 3 a 5anos) & $\begin{array}{l}\text { Transtorno do } \\
\text { Espectro Autista } \\
\text { (TEA) }\end{array}$ \\
\hline $\begin{array}{l}\text { JAARSVELD et al, } \\
2012^{21}\end{array}$ & $\begin{array}{l}\text { (775 Crianças - } 4 \text { a } 8 \\
\text { anos) }\end{array}$ & $\begin{array}{l}\text { Disfunção de } \\
\text { integração sensorial }\end{array}$ \\
\hline AVANZINO L, $2015^{22}$ & Não especificada & Distonia focal \\
\hline PARHAM LD, $2011^{23}$ & Crianças e adultos & $\begin{array}{l}\text { Desordens } \\
\text { somatossensoriais }\end{array}$ \\
\hline
\end{tabular}

A Tabela 2 contém informações sobre os desfechos correlacionando aos objetivos do artigo, dando ênfase aos benefícios alcançados em cada caso. Todos os 10 artigos constantes nesta tabela apresentam resultados positivos sobre a integração sensorial, sendo relatados nos estudos de PAVÃO S.L., ROCHA, N. A. C. F (2016) ${ }^{15}$, PEKÇETIN S., AKI E., ÜSTÜNYURT Z., KAYIHAN H (2016) ${ }^{16} e$ PERUZZOLO, D. L., SOUZA, A. P. R (2017) ${ }^{18}$ a necessidade de mais pesquisas e investigações que gerem resultados mais conclusivos. 
Tabela 2: Principais resultados discutidos nos artigos de estudos experimentais.

\begin{tabular}{ll}
\hline Estudo & Principais resultados \\
\hline SANG HJ, JUNG-HO & Treinamento de integração sensorial em pacientes \\
L, 2016 ${ }^{14}$ & $\begin{array}{l}\text { com AVC, obtendo melhora significativa na } \\
\text { atividade muscular podendo melhorar capacidade de } \\
\text { equilíbrio. }\end{array}$
\end{tabular}

PAVÃO SL, $2016^{15}$ Um conhecimento adequado dos déficits de processamento sensorial em crianças com PC permite que os profissionais envolvidos com a reabilitação adicionem estímulos sensoriais específicos em suas terapias, a fim de melhorar a independência e funcionalidade.

PEKÇETIN S, 2011 ${ }^{16}$ É necessário avaliar os recém-nascidos prematuros para SPD e, em seguida, planejar e implementar programas de intervenção de integração sensorial individualmente.

WEINSTEIN M, $2015^{17}$

São fornecidas evidências pela exploração e alteração da neuroplasticidade após a terapia intensiva bimanual com mão-braço (HABIT) em crianças com hemiparesia.

PERUZOLLO DL, $2017^{18}$

Propõe uma Intervenção Precoce a partir de referenciais psicomotores que identificam a origem precoce de sinais ou sintomas de atraso no desenvolvimento.

IWANAGA R, et al, $2013^{19}$

A Terapia de Integração Sensorial foi mais efetiva para habilidades de coordenação motora, cognitivo não-verbal, e habilidades combinadas do sistema motor sensorial e cognição em crianças com Transtorno do Espectro do Autismo.

KARIM AEA, MOHAMMED AH, $2015^{20}$

Melhora significativa das atividades motoras de crianças autistas após terapia de integração sensorial e os resultados também foram positivos para crianças com atraso no desenvolvimento motor.

JAARSVELD AV, MAILLOUX Z, HERZERG DS, $2012^{21}$

12 dos 17 critérios dos testes de integração sensorial e Praxis identificaram disfunções sensoriais em crianças norte-americanas, mas 5 testes em que as crianças africanas obtiveram resultados mais positivos do que as norte americanas, o que indica crianças que têm disfunções de integração sensorial que não foram identificados pelos testes de integração sensorial e Praxis.

AVANZINO L, $2015^{22}$

Quando há comprometimento no processamento sensorial, também o motor é colocado em disfunção. Em pacientes com distonia focal, há déficit de processamento para o planejamento e execução de movimentos.

PARHAM LD, 2011 23 A medida de fidelidade de integração sensorial Ayres tem validade de conteúdo forte. A seção de processo é confiável e válida quando avaliada por avaliadores treinados com experiência em ASI.

Fonte: próprios autores, 2021.

\section{DISCUSSÃO}

Entre os artigos obtidos, 15 têm como abordagem principal a neuroplasticidade relacionada à integração sensorial para fins terapêuticos. Destes, 6 estudos (FERNÁNDEZ MARTÍNEZ, ELIZABETH et al (2013) ${ }^{24}$, GARCÉS-VIEIRA MV, SUÁREZ-ESCUDERO JC (2014)², CECCARELLI et al $(2020)^{25}$, JULIÁN CARVAJAL-

\section{CASTRILLÓN (2013) ${ }^{26}$, KHAN F, AMATYA B, GALEA} MP, GONZENBACH R, KESSELRING J (2017) $)^{27} e$ LÓPEZ ROA LM $(2012)^{l}$ se complementam ao indicar que as terapias físicas, representadas por exercícios de estimulação sensório-motora, assim como atividades que geram experiências ambientais e excitam a memória, são capazes de trabalhar a neuroplasticidade oferecendo respostas clínicas favoráveis a cada caso. Outros 3 trabalhos (NOVAK, I., et al $(2017)^{28}$, ISMAIL F.Y.; FATEMI A.; JOHNSTON M.V. $(2017)^{4}$ e CICCHETTI D. $\left.(2015)^{3}\right)$, reforçam que os melhores resultados são obtidos quando tais intervenções terapêuticas são aplicadas precocemente em janelas oportunas ou períodos sensíveis na vida dos pacientes.

As explanações bioquímicas e fisiológicas do fenômeno da plasticidade neuronal são apresentadas mais especificamente por dois estudos, o de HOLTMAAT A.; CARONI P $(2016)^{29}$ - e o de GARCÉS-VIEIRA MV, SUÁREZ-ESCUDERO JC $(2014)^{2}$, descrevendo os processos que ocorrem ao nível celular para o pleno funcionamento da neuroplasticidade com base nas atividades empregadas. Ainda entre os estudos sobre esse tema, o papel da neuroplasticidade como neurorreabilitação em patologias cerebrais como paralisia, ataxia e AVE é abordado nos artigos de FERNÁNDEZ MARTÍNEZ, ELIZABETH et al $(2013)^{24 ;}$ NOVAK, I., et al (2017) ${ }^{28}$; FILIPPO, et al. $(2015)^{30}$; REID, L. B. et al. $(2015)^{31}$.

As remodelações neuronais, ou neuroplasticidade, são facilitadas pelas condições do meio e, por isso, um ambiente enriquecido tem maior impacto na associação do estímulo sensorial e desenvolvimento motor, assim como a intervenção de maneira lúdica, quando dirigida a crianças, cria os "desafios certos" diante da flexibilidade da entrada sensorial e simplifica as interações do indivíduo com o ambiente $^{46}$. Intervir antes mesmo da evolução das patologias e seus respectivos sintomas contribui para uma neurorreabilitação mais efetiva, que além disso deve ser inclusiva, participativa e interdisciplinar, combinando elementos teóricos e práticos para valer-se da capacidade de modulação do sistema nervoso ${ }^{32}$.

Tendo em vista a integração sensorial, foram encontrados 9 estudos sobre sua realização terapêutica. Destes, 2 artigos são relatos de casos que descrevem alguns resultados do uso da terapia. SCHAAF, R. C., HUNT, J., \& BENEVIDES, T. $(2012)^{33}$ relata melhorias das habilidades motoras e comportamentais e SNIEZYK, C. J., \& ZANE, T. L. $(2015)^{34}$ não há resultados positivos comprovadamente relacionados à terapia, o grupo populacional de ambos artigos foram pacientes autistas. Esses benefícios das habilidades motoras e comportamentais são reforçados pelo estudo 
realizado por MAY-BENSON, T. A., \& KOOMAR, J. A. $(2010)^{35}$ nos quais os ganhos são mais significativos no condicionamento motor e costumam ser sustentado por período de tempo. Contudo, 2 estudos (LEONG, H. M., CARTER, M., \& STEPHENSON, J. $(2015)^{36}$ e POLATAJKO, H. J., CANTIN, N. $(2010)^{37}$ ) defendem a carência de pesquisas sobre o tema, havendo heterogeneidade entre eles, o que gera pouca clareza nos resultados, assim é provável que essa seja a justificativa para as diferenças entre os resultados encontrados. Além disso, 3 estudos (JOHNSON MH, JONES EJH, GLIGA T. $(2015)^{38}$, ZILLI, F; LIMA, CBA; KOHLER, MC (2014) ${ }^{39}$ e REID, LB et al (2015) ${ }^{31}$ ) apresentam alguns modelos terapêuticos com suas respectivas aplicações e seus resultados clínicos embasados na capacidade adaptativa do cérebro, sendo notável que quando o foco é o treinamento motor e é feito estímulo ao desenvolvimento cerebral graças à integração sensorial, resulta em melhorias na qualidade de vida dos pacientes.

Dos artigos analisados, somente 3 discutiam diretamente sobre o Método Padovan. Desses, 2 estudos descrevem os exercícios aplicados, PADOVAN, B.A.E (1994 e 1997) ${ }^{11,12}$, um dos primeiros artigos sobre o método e BENDER, NA (2017) $)^{13}$. Há também estudos observacionais, como o de PEREIRA, LM et al $(2015)^{40}$ que apresenta os benefícios da aplicação do método sobre pacientes com dificuldades de sucção, como prematuros, hipoxiados, bebês com síndromes, tendo eles obtido a redução na dificuldade respiratória, melhora na sucção e deglutição e diminuição do período de internamento hospitalar. Também estudos observacionais instituindo o Método Padovan ${ }^{\circledR}$ como terapia de reabilitação para crianças com Síndrome alcoólica fetal ${ }^{41}$, Síndrome de TreacherCollins $^{42}$ e microcefalia pela Síndrome Congênita do Zika vírus $^{43}$, onde os autores observaram melhorias no estabelecimento de sucção, na disfagia, possibilidade de redução de broncoaspiração e aquisição dos marcos do desenvolvimento.

No Método Padovan ${ }^{\circledR}$, a criadora usou como base os conceitos de neuroplasticidade aprofundados nos estudos sobre Reorganização neurológica e funções reflexovegetativas, sendo visto resultados melhores e mais rápidos quando o foco era o uso da sequência do desenvolvimento neurológico, com execução dos movimentos básicos, que em crianças com lesões cerebrais, não conseguiam realizar ${ }^{11,12}$. Seu sistema terapêutico abrange uma atenção multissensorial, pois habilita tanto a fala, como a coordenação motora, percepção auditiva, visão e sistema vestibular pela diversidade dos exercícios ${ }^{13}$. Essa diversidade advém da base de padrões do desenvolvimento neural, resultante dos fatores genéticos e interação com ambiente, principalmente exercidos na primeira infância ${ }^{13}$.

A integração sensorial pode ser caracterizada como multissensorial além de condicionar diversos processos cognitivos. Vários sistemas sensoriais que compõem a organização neurológica concedem as mais variadas percepções sobre o meio ambiente de acordo com as interpretações de cada sentido. Alguns estímulos podem excitar todos os sentidos simultaneamente, conferindo experiências multissensoriais cujas combinações, ao longo do tempo, transformam-se em respostas adaptativas, assim como um único evento é capaz de despertar a integração de diferentes campos sensoriais do cérebro. A partir disso, a combinação de mais de um tipo de modalidade sensorial pode diminuir o tempo de resposta ao estímulo, melhorar a precisão para determinados alvos e otimizar a aprendizagem ${ }^{44}$. Visto que as desordens de processamento neurossensorial podem repercutir logo na infância e, posteriormente, na vida adulta, é essencial diagnosticar tais condições e intervir precocemente para reverter ou minimizar possíveis danos, utilizando as janelas de oportunidades. É na fase de lactente que são percebidas as informações sensóriomotoras dos cinco sentidos como tato, visão, audição, olfato e paladar, assim como também são interpretadas a posição e a movimentação do próprio corpo ${ }^{45}$.

Os primeiros exercícios do método são para atuação no corpo, onde se trabalha movimentos de braços, pernas e cabeça, integrando as cadeias musculares e que são tipos de movimentos reflexos naturais ao nascimento, movimentos que simulam o neurodesenvolvimento como rolar, rastejar, engatinhar, agachar e levantar e andar, os quais, são movimentos que fazem parte do programa genético do neurodesenvolvimento e que segue a linha das pesquisas que evidenciam a importância de terapias que estimulam as habilidades motoras e contribuem com as capacidades de coordenação motora grossa, fina e comportamento ${ }^{11,12,25}$. Ainda PADOVAN (1994) ${ }^{12}$ cita outros exercícios que são utilizados de acordo com a capacidade do paciente, a cambalhota (uso de movimentos motores), rede giratória (atua no sistema vestibular), exercícios visuais (estimula reflexos fotomotores), exercício de coordenação visomanual-motora (uso da visão e tato), pular corda (trabalhar a orientação temporo-espacial). Nos exercícios do teste de integração sensorial de Ayres, o foco se assemelha, as práticas são voltadas ao trabalho dos sentidos, é exercitado a visão (imitação de posturas), tato (uso de estímulos táteis, terapia do toque), utilização de objetos, como saco de boxe e bolas, para trabalhar equilíbrio e sistema vestibular, comparado ao do Padovan ${ }^{\circledR}$ é visível a ligação dos métodos, 
uma das grandes semelhanças está no uso de canções, todos dois utilizam durante as práticas, com objetivo de desenvolver a percepção auditiva. ${ }^{6,46,47}$ Quando comparados com os estudos de ELBASAN B, KAYIHAN H, DUSGUN I $(2012)^{46}$ e WAN YUNUS, F., et al $(2015)^{47}$, que apontam alguns exercícios da terapia de integração sensorial, é possível ver a semelhança entre as práticas e o embasamento comum que é a neuroplasticidade, reafirmando o desenvolvimento da integração sensorial pelo Método Padovan ${ }^{\circledR}$.

Em seguida, são iniciados os exercícios para funções reflexo-vegetativas: respiração, sucção, mastigação e deglutição, de caráter fundamental para sobrevivência e para a fala, e que comungam da mesma neuromusculatura, sendo, através dos exercícios executados estimulados os doze pares de nervos cranianos ${ }^{13}$. Nessa etapa é preciso harmonizar as quatro funções orais pois existe uma comunicação inerente dos músculos, o paciente tem um tratamento do seu todo, por isso os reflexos dessa terapia atingem desde da fala à postura do corpo, coordenação de movimentos e funções prélinguísticas ${ }^{12,13}$.

Em vista dessa base na neuroplasticidade da terapia do Método Padovan ${ }^{\circledR}$, seus resultados abrangem desde bebês a adultos de forma a ter capacidade curativa e preventiva, assim como na infância há maior influência de estímulos, pois, seu início precoce aumentam as chances de cura e melhores respostas, principalmente nos casos de comprometimento neurológico, pois os bebês têm dificuldades para se alimentar e manter o crescimento corporal, reduzindo sua sobrevida ${ }^{13,40}$. Segundo Buffone, Eickman e Lima (2016 ${ }^{45}$, os bebês prematuros e que tiveram hipóxia, possuem déficit significativos dos processos sensoriais que alteram o desenvolvimento neuropsicomotor e com estímulos recebidos durante o período na UTI modificam as funções motoras e da autorregulação, logo, como o visto pelo procedimentos relatados por Pereira $(2015)^{40}$, o Padovan ${ }^{\circledR}$ atuando desde a UTI faz diferença significativa para o futuro dessas crianças, como uma opção de tratamento para casos semelhantes.

Assim, modelos de terapia de reabilitação com abordagem integrativa têm sido escolhidos para o manejo de crianças e adolescentes com dificuldades de integração e processamento sensorial, como os que apresentam Transtorno do Espectro Autista (TEA), Transtorno de Déficit de Atenção e Hiperatividade (TDAH) e dificuldades de aprendizagem, de modo que a escolha do método é fundamental para o seguimento e prognóstico e a escolha do tratamento pelos terapeutas deve ser baseada em evidências ${ }^{8,9,37}$.
Associando esse estudo aos de PERUZZOLO D.L., SOUZAB A.P.R. $(2017)^{18}$ que traz técnicas para desenvolvimento psicomotor em período mais precoce pelos seus resultados positivos, mostrando a importância de intervenções precoces a partir de referenciais motores já conhecidos e AVANZINO L (2015) ${ }^{22}$ sobre a modulação das entradas sensoriais contribuindo com a organização da função motora, vê-se as vantagens do uso do Método Padovan nas populações de crianças.

\section{CONCLUSÃO}

Diante do exposto, observamos que há uma organização do Sistema Nervoso Central ao longo do desenvolvimento humano e que para isso é fundamental estímulos sensoriais externos, que nos chega através dos sentidos, sendo necessário uma integração dessas informações sensoriais que são dependentes da integridade dos circuitos cerebrais e da neuroplasticidade e que, portanto, terapias que tenham como base a integração sensório-motora se beneficiarão dessa neuroplasticidade para seus benefícios na recuperação de distúrbios do neurodesenvolvimento.

O Método Padovan ${ }^{\circledR}$, como demonstrado, é uma abordagem terapêutica sensório-motora e trabalha essa integração sensorial, dentro dos preceitos da ontogênese, tendo como fenômeno facilitador a neuroplasticidade, podendo ser incluído nos conceitos de terapia de integração sensorial. Assim, torna-se relevante desenvolver estudos científicos sobre o uso do método em pacientes com disfunções neurológicas, atentando-se para a importância da intervenção precoce a fim de amenizar possíveis repercussões durante o desenvolvimento infantil e até na vida adulta.

\section{REFERÊNCIAS}

1-López Roa LM. Neuroplasticidad y sus implicaciones en la rehabilitación. Rev Univ. salud. 2012; 14(2):197 - 204

2-Garcés-Vieira MV, Suárez-Escudero JC. Neuroplasticidad: aspectos bioquímicos y neurofisiológicos. Rev CES Med 2014; 28(1): 119-132.

3-Cicchetti D. Neural plasticity, sensitive periods, and psychopathology. Development and Psychopathology. Cambridge University Press; 2015;27(2):319-20. DOI: https://doi.org/10.1017/S0954579415000012

4-Ismail F.Y., Fatemi A., Johnston M.V. (2017). Cerebral plasticity: Windows of opportunity in the developing brain European Journal of Paediatric Neurology 2016 Aug. 21(1) 23-48. DOI: https://doi.org/10.1016/j.ejpn.2016.07.007

5-Costa, M.A.O. Qualidade da integração sensorial e organização dos comportamentos de vinculação na criança. Porto. Dissertação de Mestrado em Psicologia - Faculdade de Psicologia e de Ciências da Educação, Universidade do Porto; 2000 
6- Tudela-Torras M, Abad-Mas L, Tudela-Torras E. Integración sensorial: beneficios y efectividad del abordaje terapéutico en los trastornos del procesamiento sensorial. Rev Neurol 2017; 64 (Supl 1):S73-7.

7-Schaaf, R. C. and Miller, L. J. Occupational therapy using a sensory integrative approach for children with developmental disabilities. Ment Retard. Dev. Disabil. Res. Rev., 11: 143-148, 2005.

8-Camarata S, Miller LJ and Wallace MT. Evaluating Sensory

Integration/Sensory Processing Treatment: Issues and Analysis. Front. Integr. Neurosci. 14:556660. doi: 10.3389/fnint.2020.556660.

9-Kilroy E, Aziz-Zadeh L and Cermak S. Ayres Theories of Autism and Sensory Integration Revisited: What Contemporary

Neuroscience Has to Say. Brain Sci. 2019, 9, 68; doi: $10.3390 /$ brainsci9030068

10- Pereira, LM. Padovan Method as Early Stimulation in Neonatal Intensive Care Unit [editorial]. Int arc med 2015; 8(166):1-3.

11-Padovan B.A.E. Reorganização Neurofuncional - Método Padovan. Jornal Brasileiro de Ortodontia e Ortopedia Maxilar 1997 julho/agosto. v2; n10.

12- Padovan, B.A.E. Reorganização Neurofuncional - Método Padovan. Revista Temas sobre Desenvolvimento 1994 abril; 3(17) 1-11.

13-Bender, NA. TMF no Método Padovan ${ }^{\circledR}$ Reorganização Neurofuncional (4) 1: e2017-09; doi: 10,14620 / stadbs 171109

14- Sang HJ, Jung-Ho L. Impact of sensory integration training on balance among stroke patients: Sensory integration training on balance among stroke patients. Open Med 2016 aug; 11(1): 330-335.

15-Pavão S.L., Rocha, N. A. C. F. Sensory processing disorders in children with cerebral palsy. Infant Behav Dev 2016 Nov. 46 (2017) 1-6.

16-Pekçetin S., Akı E., Üstünyurt Z., Kayıhan H. The Efficiency of Sensory Integration Interventions in Preterm Infants. Epub 2016 Aug. 123(2) 411-423.

17-Maya Weinstein, Vicki Myers, Dido Green, et al., "Brain Plasticity following Intensive Bimanual Therapy in Children with Hemiparesis: Preliminary Evidence,” Neural Plasticity, vol. 2015, Article ID 798481, 13 pages, 2015. DOI: https://doi.org/10.1155/2015/798481.

18-Peruzzolo, D. L., Souza, A. P. R de. Uma hipótese de funcionamento psicomotor como estratégia clínica para o tratamento de bebês em intervenção precoce. Cad. Bras. Ter. Ocup. 2017. 25(2) 427-434. DOI:

http://dx.doi.org/10.4322/0104-4931.ctoEN0864.

19-Iwanaga, R., Honda, S., Nakane, H., Tanaka, K., Toeda, H., \& Tanaka, G. (2014). Pilot study: Efficacy of sensory integration therapy for Japanese children with high-functioning autism spectrum disorder. Occupational Therapy International, 21, 4-11. DOI:10.1002/ oti.1357.

20-Karim A.E.A., Mohammed A.H. Effectiveness of sensory integration program in motor skills in children with autism. Egypt J Med Hum Genet 2015; 16 (4), 375-380. DOI: 10.1016/i.ejmhg.2014.12.008

21-Jaarsveld Annamarie Van, Mailloux Zoe, Herzberg David S. The use of the Sensory Integration and Praxis Tests with South African children. S. Afr. j. occup. ther. [Internet]. 2012; 42(3): 12-18.

22-Avanzino, L., Tinazzy, M., Lonta, S., Fiorio, M. Sensory-motor integration in focal dystonia. Infant Behav Dev December 2015. 79 (2015) 288-300.

23-Parham, L. D., Roley, S. S., May-Benson, T. A., Koomar, J., Brett-Green, B., Burke, J. P., et al. (2011). Development of a fidelity measure for research on the effectiveness of the Ayres Sensory integration intervention. American Journal of Occupational Therapy, 65, 133-142.
24-Fernández Martínez, Elizabeth et al. La neurorrehabilitación como alternativa esencial en el abordaje terapéutico de las ataxias cerebelosas. Rev Cubana Salud Pública 2013 Set. 39(3): 489-500.

25-Ceccarelli SB, Ferrante C, Gazzola E, Marzocchi GM, Nobile M, Molteni $\mathrm{M}$ and Crippa A. Fundamental Motor Skills Intervention for Children with Autism Spectrum Disorder: A 10-Year Narrative Review. Children 2020, 7 , 250; doi:10.3390/children7110250.

26-Julián Carvajal-Castrillón. Neuropsychological rehabilitation in the acute phase of acquired brain injury. Acta Neurol Colomb 2013;29:173-179.

27-Khan F, Amatya B, Galea MP, Gonzenbach R, Kesselring J.

Neurorehabilitation: applied neuroplasticity. J Neurol. 2017 Mar; 264(3):603615. DOI: $10.1007 / \mathrm{s} 00415-016-8307-9$

28-Novak, I., Morgan, C., Adde, L., Blackman, J., Boyd, RN, BrunstromHernandez, J., et al. (2017). Early, accurate diagnosis and early intervention in cerebral palsy: Advances in diagnosis and treatment. JAMA Pediatrics, 171(9), 897-907. DOI: http://dx.doi.org/10.1001/jamapediatrics.2017.1689

29-Holtmaat, A., Caroni, P. Functional and structural underpinnings of neuronal assembly formation in learning. Nature Neurosciense 2016 oct. 19 $1553-1562$

30-FILIPPO, TRM et al. Neuroplasticidade e recuperação funcional na reabilitação pós-acidente vascular encefálico. Acta Fisiátrica 2015 jun. 22(2) 93-96. DOI: http://dx.doi.org/10.5935/0104-7795.20150018

31-Reid, L. B. et al. Rehabilitation and neuroplasticity in children with unilateral cerebral palsy. Nat. Rev. Neurol. 2015 Jun; 11 390-400. DOI: 10.1038/nrneurol.2015.97

32-Lane, S J, Schaaf, R S. Examining the Neuroscience Evidence for SensoryDriven Neuroplasticity: Implications for Sensory-Based Occupational Therapy for Children and Adolescents. May/June 2010. 64 (3): 375-390. doi: 10.5014/ajot.2010.09069.

33-Schaaf, R. C., Hunt, J., Benevides, T. Occupational therapy using sensory integration to improve participation of a child with autism: A case report. American Journal of Occupational Therapy 2012; 66, 547-555. DOI http://dx.doi.org/10.5014/ajot.2012.004473

34-Sniezyk, C. J.; Zane, T. L. Investigating the effects of sensory integration therapy in decreasing stereotypy. Focus on Autism and Other Developmental Disabilities 2015; 30(1), 13-22. DOI: http://dx.doi.org/10.1177/1088357614525663

35-May-benson, T. A., Koomar, J. A. (2010). Systematic review of the research evidence examining the effectiveness of interventions using a sensory integrative approach for children. American Journal of Occupational Therapy 2010; 64, 403-414. DOI: 10.5014/ajot.2010.09071

36-Leong, H. M., Carter, M.; Stephenson, J. Systematic review of sensory integration therapy for individuals with disabilities: Single case design studies. Research in Developmental Disabilities 2015; 47, 334-351.

37-Polatajko, H. J., Cantin, N. Exploring the effectiveness of occupational therapy interventions, other than the sensory integration approach, with children and adolescents experiencing difficulty processing and integrating sensory information. American Journal of Occupational Therapy, 2010; 64 , 415-429. DOI: 10.5014/ajot.2010.09072.

38-Johnson MH, Jones EJH, Gliga T. Brain adaptation and alternative developmental trajectories. Development and Psychopathology. Cambridge University Press; 2015;27(2):425-42. DOI:

https://doi.org/10.1017/S0954579415000073

39-Zilli, F; Lima, CBA de; Kohler, MC. Neuroplasticidade na reabilitação de pacientes acometidos por AVC espástico. Revista de Terapia Ocupacional da Universidade de São Paulo 2014 Dec. 25(3) 317-322.

DOIhttp://dx.doi.org/10.11606/issn.2238-6149.v25i3p317-322 
40- Pereira, LM; et al. Padovan Method of Neurofunctional Reorganization As a Way for Neurological Recovery in Newborns. International Archives of Medicine, 2015, sep. V:8. DOI: http://dx.doi.org/10.3823/1829.

41-Pereira LM, Vileicar DC, Uchôa MMA. Neurorreabilitação com o método Padovan em recém-nascidos com síndrome alcoólica fetal: relato de 2 casos. J Health Biol Sci. 2018 Abr-Jun; 6(2):214-216.

42-Delmondes, EL; Albuquerque, LTC de; Pereira, LM. Neurorehabilitation with Padovan Method in a Newborn with Treacher Collins Syndrome: A Case Report. Am. In. Mult. J., Oct. 2018 (5) 3, 01-07.

43-Meneses MIN et al. Avaliação dos efeitos do método Padovan® no desenvolvimento

neuropsicomotor de crianças com microcefalia: série de casos. Revista Eletrônica Acervo Saúde / Electronic Journal Collection Health | ISSN 2178 2091. Out 2019. Vol.Sup.34 | e1509 | DOI: https://doi.org/10.25248/reas.e1509.2019

44-Dionne-Dostie E, Paquette N, Lassonde M, Gallagher A. Multisensory Integration and Child Neurodevelopment. Brain Sciences [Internet]. MDPI AG; 2015 Feb 11;5(1):32-57. DOI: http://dx.doi.org/10.3390/brainsci5010032

45-Buffone, FRG, Eickman, SH, Lima, MC. Processamento sensorial e desenvolvimento cognitivo de lactentes nascidos pré-termo e a termo. Cad. Ter. Ocup. UFSCar, São Carlos 2016, 24 (4): 695-703. DOI: http://dx.doi.org/10.4322/0104-4931.ctoAO0731

46-Elbasan B, Kayihan H, Dusgun I. Sensory integration and activities of daily living in children with developmental coordination disorder. Ital J Pediatr 2012; 38:14. DOI: $10.1186 / 1824-7288-38-14$.

47-Wan Yunus, F., Liu, K. P., Bissett, M., \& Penkala, S. Sensory-based intervention for children with behavioral problems: A systematic review. Journal of Autism and Developmental Disorders 2015; 45(11), 3565-3579. 